\title{
Estructura territorial del turismo astronómico en la región de Coquimbo, Chile
}

\author{
Territorial structure of astronomical tourism in the region of \\ Coquimbo, Chile
}

\author{
Juan de Dios Páramo Gómez ${ }^{1}$ \\ Álvaro Sánchez Crispín ${ }^{2}$ \\ Universidad Nacional Autónoma de México, México
}

\begin{abstract}
Resumen
El turismo astronómico es parte del turismo de intereses especiales, esta actividad tiene la particularidad de ser practicado en lugares muy puntuales en el mundo que reúnen características como latitud, altitud, clima, calidad de la atmósfera, que permiten la observación directa de la bóveda celeste. La región de Coquimbo, localizada en el Norte Chico de Chile, es un sitio privilegiado para la práctica del turismo astronómico, tanto por su posición en el globo terráqueo como por su clima árido dominante, a lo que se suma la disponibilidad de infraestructura de transporte y alojamiento, y la existencia de observatorios (científicos y turísticos). Este trabajo tiene como finalidad revelar la estructura territorial que ha conformado el turismo astronómico en esta parte de Chile, y expone la importancia del núcleo central en la actividad turística regional de la conurbación La Serena-Coquimbo.
\end{abstract}

Palabras clave: Turismo de intereses especiales, turismo astronómico, estructura territorial, Coquimbo, Chile

1 Posgrado en Geografía, Instituto de Geografía, Universidad Nacional Autónoma de México, Circuito de la Investigación Científica, 04510, Coyoacán, México, CDMX. E-mail: juandedios_p9j7@hotmail.com

2 Departamento de Geografía Económica, Instituto de Geografía, Universidad Nacional Autónoma de México, Circuito de la Investigación Científica, 04510, Coyoacán, México, CDMX. E-mail: asc@igg.unam.mx

Este artículo corresponde a la ponencia presentada en el 35th Conference of Latin American Geographers realizada en San José, Costa Rica del 20 al 22 de mayo del 2018. 
Juan de Dios Páramo Gómez, Álvaro Sánchez Crispín. Estructura territorial del turismo astronómico en la región de Coquimbo, Chile

\begin{abstract}
Astronomical tourism, a segment of the tourism of special interests, made its debut in the international arena just a couple of decades ago. This type of tourism can only be practiced in certain areas of the world where latitude, altitude, climate and the quality of atmosphere contribute to generate the natural conditions specifically needed to observe the celestial dome. The Norte Chico in Chile is a privileged region in this respect, since its relative location on Earth, the dominant dry climate, as well as the infrastructure available and accessibility to observatories (scientific or otherwise) has given way to the expansion of astronomical tourism in several locations within the Coquimbo region, a part of Norte Chico. This paper deals with the issue of territorial structure, a geographical fundamental concept, useful to explaining the expansion of astronomical tourism in this part of Chile. In doing so, nuclei, flows and surfaces created by this type of tourism economy are revealed.
\end{abstract}

Keywords: Special interest tourism, astronomical tourism, territorial structure, Coquimbo, Chile

\title{
Introducción
}

De acuerdo con Barrientos y López (2008) para la instalación de observatorios astronómicos, tanto de índole científica como turística, se requiere, sin duda alguna, una nula presencia de contaminación lumínica, que se refiere principalmente a la emisión, reflexión y difusión de luz artificial en los gases y partículas de la atmósfera; la principal causa de este fenómeno es el número y tipo de luminarias que se concentran en un sitio, principalmente asociado con lugares donde se ubican los grandes centros urbanos. Éstos se pueden identificar, en el planeta, a través de un mapa del cielo nocturno donde se concentran los lugares con total oscuridad y condiciones idóneas necesarias para contemplar, estudiar y valorar el firmamento; sin embargo, aunque se observen lugares sin este tipo de contaminación en diversas partes del mundo, son pocos los países y los sitios que logran reunir las características geográficas y astronómicas esenciales para apreciar los fenómenos que acontecen en el cielo. Es por ello que el hemisferio sur y el norte de Chile se consideran ideales para la construcción de los observatorios astronómicos más grandes e importantes a nivel mundial (OPCC, s/a; Viterbo, 2012).

El interés del ser humano por tratar de interpretar lo que se ve en el cielo, desde la antigüedad hasta el presente, explica la construcción de instalaciones, herramientas y aparatos que ayudan a contemplar la bóveda celeste; esta infraestructura ha logrado evolucionar con el tiempo y, actualmente, la observación del cielo con fines astronómicos no se limita a su estudio, también comprende la contemplación de este recurso por parte de los turistas. Según autores como Barraza y González, 2012; Viterbo, op.cit., y el Programa de Mejoramiento para la Competitividad (PMC), 
2008, estos lugares se encuentran principalmente en el hemisferio sur; ejemplo de ello son el Norte Grande y el Norte Chico de Chile, Argentina, Suráfrica, Australia y Nueva Zelandia. Otros lugares ideales para realizar observación astronómica son Hawaii y Arizona (Estados Unidos), Islas Canarias (España), Italia y el norte de México.

La región de Coquimbo, también llamada IV Región, ubicada en el norte de Chile, posee condiciones geográfico-físicas favorables para la investigación y observación astronómica. Los factores naturales que se presentan en este lugar se asocian con su ubicación en el hemisferio sur que privilegia la observación de un mayor número de fenómenos astronómicos, una alta frecuencia de noches fotométricas; una nula contaminación lumínica; un relieve de gran altitud e, igualmente, el clima, que presenta poca precipitación y humedad relativa muy baja durante el año. Además, esta región cuenta con una infraestructura de comunicación y servicios adecuada para el establecimiento de diversos observatorios astronómicos, tanto científicos como turísticos.

El turismo astronómico es una nueva vertiente del turismo contemporáneo, llamado de intereses especiales (TIE), que se centra en elementos particulares del espacio geográfico: glaciares, fauna endémica, cenotes, centros religiosos o la bóveda celeste. Es la versión opuesta del turismo de sol y playa, por no ser masivo y porque las personas que se interesan en el TIE tienen un conocimiento previo, aunque sea mínimo, de lo que van a apreciar en un determinado lugar, por muy lejos o inaccesible que éste sea, como es el caso de la atmósfera terrestre durante la noche y que contiene elementos de interés como los propios constituyentes del Sistema Solar, las nebulosas o las estrellas más brillantes del firmamento. En este trabajo se presentan los resultados de una investigación que tuvo por finalidad establecer el tramado territorial gestado por la implantación de este tipo específico de turismo en la IV Región de Chile.

Parte de la historia de la astronomía en Chile ha tenido origen en Coquimbo, con la construcción de observatorios, en los años sesenta, lo que le otorga un lugar de privilegio en la evolución de esta ciencia. En los años noventa, da inicio una nueva vertiente del turismo mundial en Chile, con la apertura de uno de los primeros observatorios turísticos en el mundo, el ubicado en el cerro Mamalluca, cercano a la localidad de Vicuña, en la provincia de Elqui, al norte del país (Ramírez, et.al., 2011). 
Juan de Dios Páramo Gómez, Álvaro Sánchez Crispín. Estructura territorial del turismo astronómico en la región de Coquimbo, Chile

\section{Turismo astronómico}

Entre los factores que explican por qué el turismo astronómico forma parte del TIE, se deben tomar en cuenta los siguientes argumentos:

- Interés. Consiste en la pretensión y particularidad del recurso turístico que quiere observar el turista, además de su percepción y conocimiento respecto a la bóveda celeste.

- Motivación. Las razones que encaminaron a la persona a realizar una visita a un sitio donde se práctica la observación astronómica científica o amateur.

- Cercanía del lugar. Al ser una actividad que no se encuentra todavía masificada, los lugares donde se realiza son muy específicos; las distancias a los sitios de pernocta son grandes y la accesibilidad a los puntos de observación suele ser difícil, porque se requieren desplazamientos de noche (sobretodo el regreso al lugar de pernocta).

- $\quad$ Promoción turística. ¿Cuál fue el medio por el que se enteró el turista que existía la observación astronómica? ¿Realmente se sabe que existe el turismo astronómico?

- $\quad$ Singularidad del destino turístico. Al considerarse un turismo no masificado, la escala del destino desempeña un papel importante; el turismo astronómico es una actividad nueva a nivel mundial, y los lugares en que los se puede llevar a cabo son puntuales y contados.

Lépez y Torre (2007) definen al turismo astronómico como “... toda actividad desplegada por las personas en los sitios astronómicos con fines recreativos, esparcimiento y de conocimiento de las instalaciones, el instrumental, los programas y proyectos de investigación vinculados al cosmos e interpretación de las leyes que lo gobiernan".

Otra definición de turismo astronómico está dada por Raymond (2013), quien considera que es una forma de turismo responsable, en un espacio natural, con infraestructura astronómica, como observatorios que posean telescopios y que, a la vez, la contemplación del cielo como recurso promueve el conocimiento y permite tener una experiencia totalmente diferente de lo habitual, del turismo masivo.

Por tanto, el turismo astronómico se puede definir como una nueva vertiente del turismo mundial en la que el firmamento funciona como recurso 
natural para su observación. Asimismo, este tipo de turismo se asocia con características particulares del entorno físico, infraestructura como telescopios u observatorios para contemplar distintos fenómenos astronómicos de interés, para que el turista tenga una experiencia de vida singular y obtenga una sensación positiva de contemplar la bóveda celeste (Figura 1).

Por otra parte, Weaber (2011) considera al turismo astronómico como "Ecoturismo celestial"; esto lo define a partir del interés de los visitantes, que se enfocan principalmente en la observación y apreciación de la ocurrencia natural de un fenómeno celeste. Además, propone que la observación de cometas, auroras boreales, la formación de nubes, atardeceres grandiosos, y cielos llenos de estrellas pueden ser considerados como recurso turístico para esta nueva vertiente del turismo. También, clasifica los distintos tipos de observación (nocturna, diurna y crepuscular), de acuerdo con el momento del día y el tipo de fenómeno que ocurra. A diferencia de otras conceptualizaciones, este autor clasifica no sólo la observación estelar de la bóveda celeste durante la noche, sino otros fenómenos astronómicos que incluyen tres bloques de observación; el primero, de manera nocturna, que contempla fenómenos comúnmente observados, pero que demanda condiciones geográfico-físicas y astronómicas particulares. En segunda y tercera instancia, la mayoría de los fenómenos se presentan en forma cotidiana, pero el impacto visual que resulta de interés para el turista, depende, en gran medida, de condiciones meteorológicas; igualmente sucede para la observación de eclipses y sol de medianoche, porque éstos obedecen primordialmente a factores astronómicos temporales. 
Juan de Dios Páramo Gómez, Álvaro Sánchez Crispín. Estructura territorial del turismo astronómico en la región de Coquimbo, Chile

Figura 1. Aspectos que caracterizan al turismo astronómico

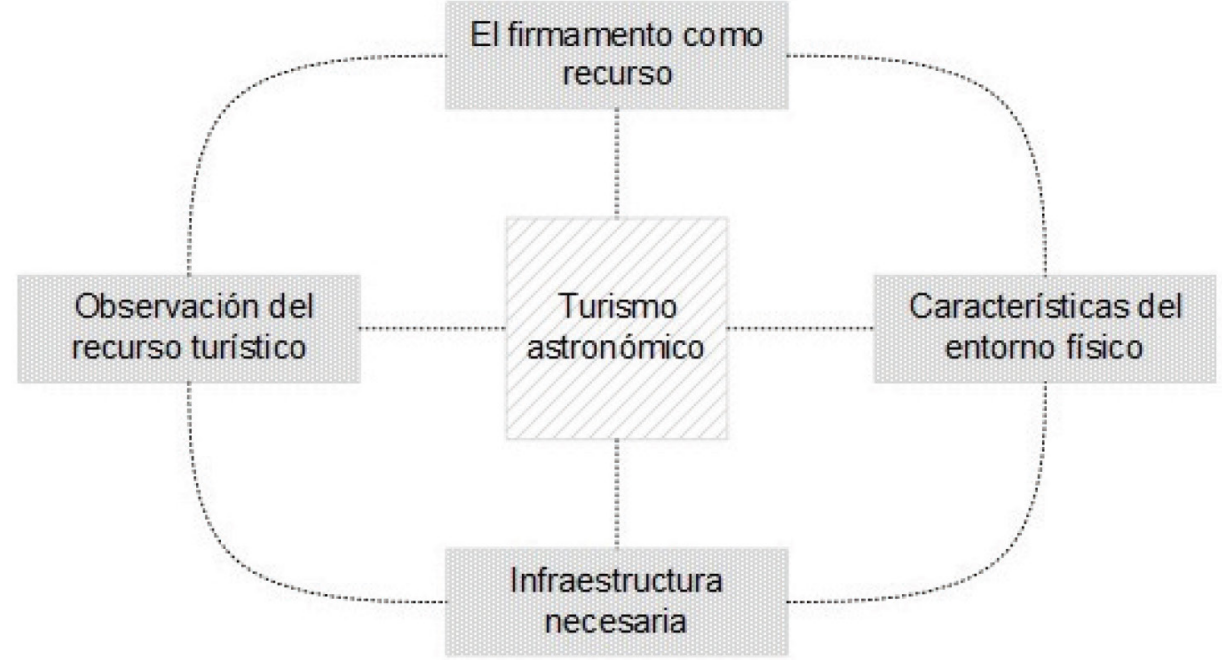

Fuente: elaborado con base en Ramírez, 2011 y Raymond, 2013.

La presente investigación está enfocada a la observación astronómica nocturna porque, en su mayoría, es la que actualmente se oferta al turista en la región de Coquimbo.

\section{Método}

En esta investigación se procedió del modo siguiente para alcanzar el objetivo central de la misma y que persigue revelar el tramado territorial del turismo astronómico en la región de Coquimbo, Chile.

Se realizó una visita a la zona de estudio, en abril y mayo de 2014, con el fin de examinar de cerca la dinámica que se produce por la presencia de los observatorios astronómicos en la región mencionada y por la promoción de visitas que se hace desde lugares como la conurbación La Serena-Coquimbo, núcleo urbano primario donde radican los principales servicios y comunicación útiles a los turistas que pretenden visitar las instalaciones de diversos observatorios de la región. En particular, se hizo el viaje nocturno al observatorio Cerro Mamalluca, cercano a la comunidad de Vicuña. En tal oportunidad, se compiló información cualitativa y cuantitativa respecto al turismo astronómico, y al turismo en general, en la IV Región. 
Posteriormente, se visitó la Universidad de La Serena para compilar información sobre el tema que interesa a esta investigación, además de sostener entrevistas con académicos, del área de Astronomía que han colaborado en la elaboración de proyectos de turismo astronómico en la región. Se realizó una revisión del material bibliográfico y estadístico disponible en impresos y otras formas de almacenamiento de información. En particular, se dispuso de material publicado por el Servicio Nacional de Turismo de Chile (SERNATUR), ofrecido en su oficina regional en La Serena. Asimismo, se tuvo acceso a publicaciones recientes, de editoriales locales, sobre turismo regional y, en particular, sobre turismo astronómico. Consecuentemente, se consultaron las distintas ofertas de agencias de viajes en La Serena con el fin de establecer los tipos asociados con el turismo astronómico que, desde ahí, se ofrecen y las opciones de acceso a los diferentes lugares donde éste es ejecutado.

Cabe señalar, que se estableció contacto con los observatorios de la zona, científicos y turísticos, para saber las modalidades de recepción de visitantes y el origen geográfico de éstos. En particular, se estableció comunicación con los observatorios Mamalluca, Pangue, Cerro Mayu y Cerro La Silla.

Una vez hecha la compilación de información, centrada en la ubicación de infraestructura especializada para la realización del turismo astronómico y la procedencia geográfica de los visitantes, se elaboró la cartografía temática correspondiente. En ella se revela el arreglo territorial contemporáneo del turismo astronómico en esta región de Chile, a través de la manifestación espacial de los recursos puntuales (cerros, cañadas, observatorios), las rutas turísticas propuestas por el gobierno chileno asociadas con la Astronomía y los nodos de articulación donde radican los servicios y las comunicaciones que vinculan el interior de la propia región de Coquimbo y con el resto de Chile, y del mundo.

\section{Contexto del turismo en la región de Coquimbo}

Las categorías del turismo a nivel mundial son numerosas, y se asocian con diversos recursos turísticos que motivan la visita por parte de distintas personas. Newsome, Moore y Dowling (2012) proponen dos grandes categorías dentro del turismo; una de ellas es el turismo de masas (sol y playa) y la otra asocia con fines e intereses más específicos por parte 
Juan de Dios Páramo Gómez, Álvaro Sánchez Crispín. Estructura territorial del turismo astronómico en la región de Coquimbo, Chile

del turista y se revela en el turismo religioso, urbano, rural, oscuro, científico y el astronómico, entre otros.

Trauer, (2006: 186) arguye que “... el término de Turismo de Intereses Especiales se compone principalmente por dos grandes indicadores: El primero "el interés especial", que sugiere considerar la necesidad del contexto del ocio, de la actividad que se desarrollará en el lugar; el segundo es "el turismo", que puntualiza en la comercialización del recurso". De acuerdo con este mismo autor y con Brotherton y Himmetoglu (1997), los intereses del turismo pueden clasificarse de tres maneras y en forma de un ciclo (Figura 2):

- Turismo de interés general: turismo convencional de masas, que ocupa gran parte del territorio de acogida y genera exceso en relación con la capacidad de carga del sitio; a la vez, la dinámica de este espacio tiende a ser similar respecto a las actividades realizadas por el turista (turismo de sol y playa).

- Turismo de interés mixto: es un turismo menos convencional; no obstante, hay un interés particular, por lo que la visita al destino turístico tendrá una menor duración y, a la vez, se realizan dinámicas específicas, distintas a las que genera el turismo de masas. La escala del sitio dependerá de la intención, ocio y tiempo libre del turista; ejemplo de ello es el turismo urbano.

- $\quad$ Turismo de intereses especiales: es totalmente contrario al turismo de masas, los lugares donde se práctica son muy puntuales y se encuentra asociado con las nuevas tendencias del turismo que tienen como objetivo central satisfacer necesidades muy particulares de los turistas que buscan, al realizar esta actividad, un cierto grado de exclusividad. 
Figura 2. Posición y elementos del turismo de interés especial

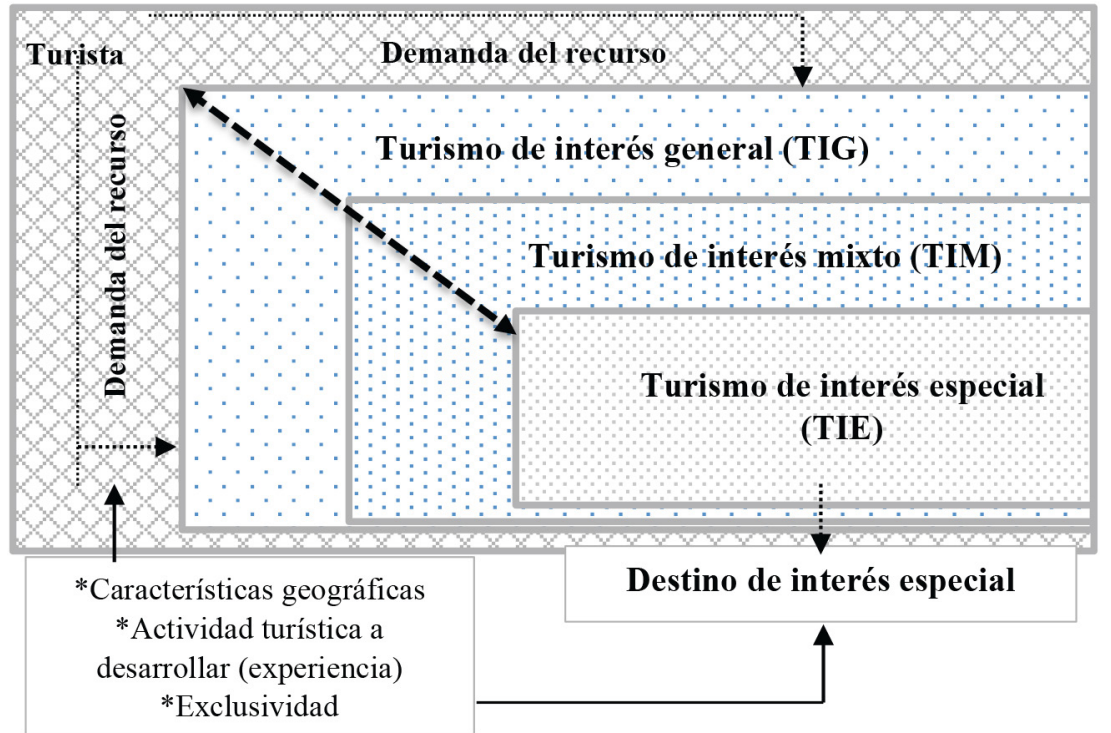

Fuente: elaborado con base en Brotherton y Himmetoglu, 1997; Trauer, 2006.

Así, se puede considerar que existe una categoría denominada Turismo de Intereses Especiales (TIE) que Espinosa, Llancaman y Sandoval, (2014: 114) identifican como: “... un tipo de turismo que está basado en el desarrollo de viajes individuales, no comercializados en forma masiva, sino que diseñados "a la medida" de cada turista. Está directamente relacionado con motivaciones turísticas de nichos especiales".

Por lo tanto, el TIE puede inferirse como un tipo del turismo, que depende del interés que tenga el turista al momento de llegar a un lugar determinado para disfrutar de algo específico.

Kruja y Gjyrezi (2011: 79) señalan que el "TIE comprende diferentes formas de interés especial que tienen los viajes, los cuales se conocen como nichos de mercado específicos que en los últimos años han ganado popularidad entre diversos actores que se encuentran dentro de la industria turística... algunos de ellos se han considerado como sostenibles y se muestran como categorías de TIE".

Entonces, el TIE se considera un turismo a pequeña escala, donde existen recursos particulares, de un interés en específico, y al cual no todo tipo de turista tiene acceso. Según Kruja y Gjyrezi (Ibíd.), el turista de 
Juan de Dios Páramo Gómez, Álvaro Sánchez Crispín. Estructura territorial del turismo astronómico en la región de Coquimbo, Chile

intereses especiales disfruta normalmente de actividades en contacto con la naturaleza, donde busca un tema totalmente diferente a lo que ofrece el turismo habitual; así, el TIE tiene como singularidad que el sujeto explore, descubra y aprenda del recurso turístico que se oferte.

\section{Relación del turismo astronómico con la Geografía}

Otra relación específica del turismo astronómico con la Geografía se asocia con la particularidad del territorio donde se desarrolla, debido a que deben existir factores astronómicos y geográfico-físicos que se concatenan para crear un vínculo que distinga al sitio donde se practica esta actividad. Algunos de los elementos característicos del lugar se resumen en su ubicación, el tipo de clima que prevalece, las condiciones meteorológicas, la "limpieza" del cielo, la altitud y el relieve.

El turismo astronómico se basa tanto en el fenómeno observado como en la dinámica turística que genera. Pocos estudios se han realizado en Geografía respecto este proceso; esta investigación establece una forma de examinar el turismo astronómico desde un enfoque geográfico ya que proporciona una visión territorial de la actividad turística en un lugar determinado, y de acuerdo con Páramo (2015) considera los puntos siguientes:

- $\quad$ La existencia de recursos naturales particulares; en este caso, la bóveda celeste.

- $\quad$ El aprovechamiento de este recurso por parte de la sociedad para hacerlo ingresar a la dinámica turística.

- $\quad$ El estudio de los flujos y relaciones generadas por esa actividad turística, con alcances geográficos a distintas escalas.

En Geografía del turismo existen procesos puntuales que vuelven particular al turismo astronómico; uno de ellos es la procedencia de los flujos turísticos y cómo se comportan dentro del territorio. En consonancia, se tiene que saber si el lugar cuenta con infraestructura necesaria para hospedaje; si existen canales de articulación por los cuales puede viajar el turista y, de manera precisa, si se ofrecen opciones de un turismo complementario, ya que el turista necesita esperar cierto tiempo para que se cumpla el objetivo principal de su visita al sitio, que es contemplar la bóveda celeste (a través de

190 Revista Geográfica de América Central No 61E (3) Especial CLAG e-ISSN 2215-2563 • Julio-diciembre 2018 • pp. 181-206 Doi: http://dx.doi.org/10.15359/rgac.61-3.10 
un telescopio) o de una manera sencilla, sin aparato alguno de observación astronómica, simplemente alzando la vista al cielo (Figura 3).

Figura 3. Factores asociados con el turismo astronómico

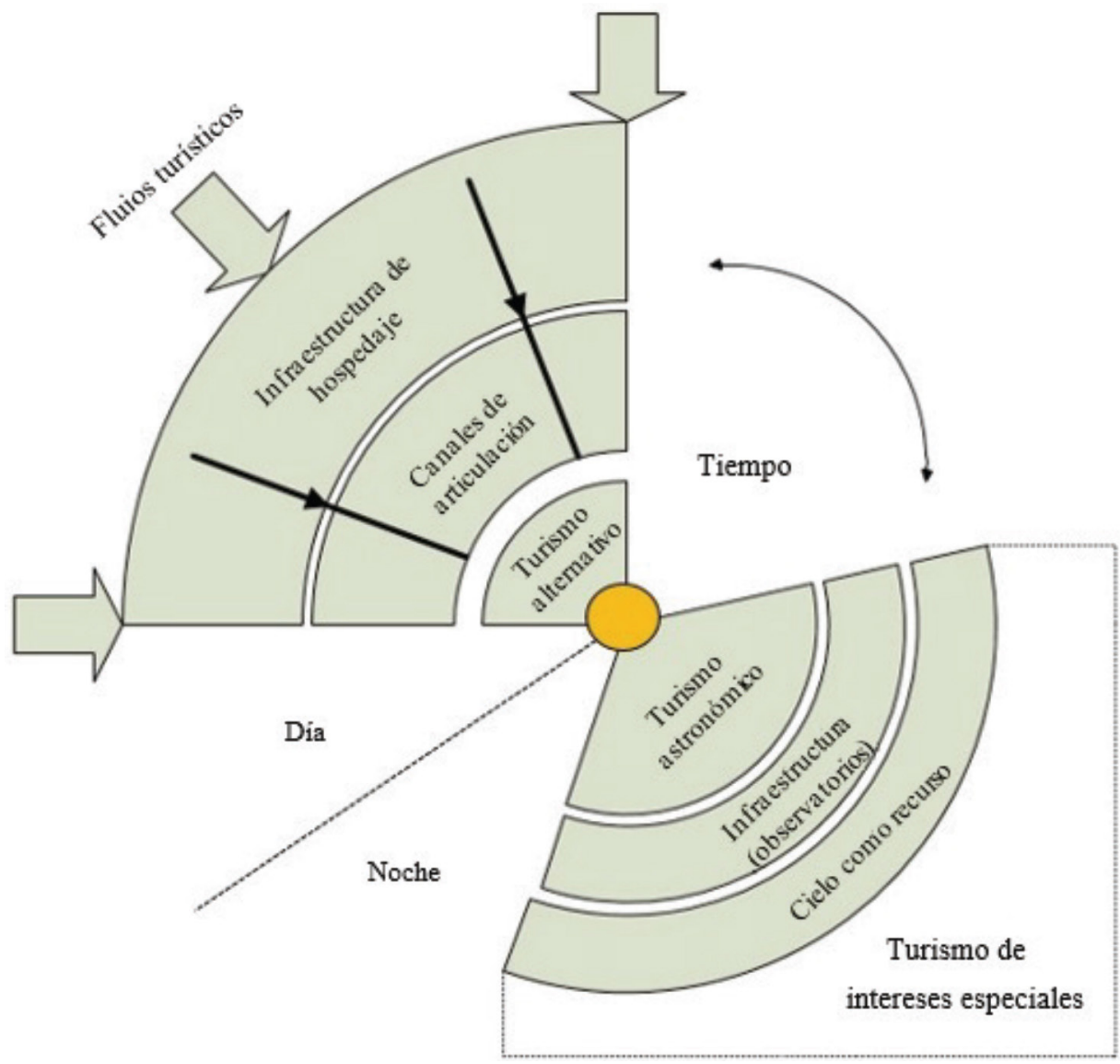

Fuente: elaborado con base en Barrado, 2004.

El turismo es una actividad que ha evolucionado paulatinamente en Chile; esto se refleja en el incremento de turistas que arriban al país en los últimos veinte años y el crecimiento de las divisas que genera, aunque la aportación del turismo al PIB es todavía incipiente con respecto a otras actividades (INE, 2011). El turismo en el país se encuentra bajo la supervisión del Servicio Nacional de Turismo (SERNATUR) que está encargado del desarrollo de planes y programas que fomenten la oferta, promoción y difusión 
Juan de Dios Páramo Gómez, Álvaro Sánchez Crispín. Estructura territorial del turismo astronómico en la región de Coquimbo, Chile

de los recursos turísticos entre los turistas nacionales y extranjeros; además, esta entidad se ha encargado de la elaboración de un catastro de "atractivos turísticos" e identificación de áreas turísticas a nivel local, regional y nacional. Éstas concentran cierto valor turístico y, de manera frecuente, son destino primordial para los turistas. El área de interés principal para el turismo astronómico es el valle del Elqui, en Coquimbo; sin embargo, existen otros lugares al norte del país que también ofertan esta actividad.

La economía de la región de Coquimbo se basa en actividades asociadas con la agricultura y la minería; la evolución del turismo como actividad económica regional es fundamental, porque ha posicionado a Coquimbo entre los principales destinos de turistas nacionales e internacionales, debido a los múltiples recursos turísticos con los que cuenta; esto ha generado actividades enfocadas a atender el turismo de sol y playa, y las vertientes de tipo religioso, cultural, enológico y astronómico, en la IV Región.

El SERNATUR ha catalogado, en la región de Coquimbo, alrededor de 300 sitios turísticos donde el mayor número de recursos se asocian con: sitios naturales (más del 30\%); elementos de folklore (23\%); y los de interés histórico y cultural (18\%), mientras que, en menor número, se hallan los lugares de realización técnica, científica o artística contemporánea con el $15 \%$, donde se posiciona el "turismo astronómico"; acontecimientos programados $(11 \%)$ y con un solo sitio $(0.3 \%)$ los centros o lugares de esparcimiento (Figura 4).

Así, se puede afirmar que en la IV Región hay un predominio de recursos turísticos asociados con sitios históricos y/o culturales, seguido de los naturales; no obstante, existen otros destinos catalogados como de realización técnica-científica que se logran posicionar a través de la oferta, principalmente, de los observatorios astronómicos tanto turísticos como científicos para su visita. Para 2010, algunos de estos recursos turísticos ofertados participaron en el concurso "Las 7 maravillas turísticas de la región de Coquimbo", elaborado por el Servicio Nacional de Turismo regional. Los resultados se publicaron en febrero de 2011 y los lugares ganadores fueron los siguientes:

1.Observatorio Cruz del Sur en Combarbalá; 2. Casco Histórico de La Serena; 3. Basílica Menor en Andacollo; 4. Reserva Nacional Las Chinchillas en Illapel; 5. Reserva Nacional Pingüino de Humboldt en La 
Higuera; 6. Parque Nacional Bosque Fray Jorge en Ovalle; 7. Los humedales de Tongoy en Coquimbo.

Figura 4. IV Región: categoría y número de recursos turísticos catalogados, 2012

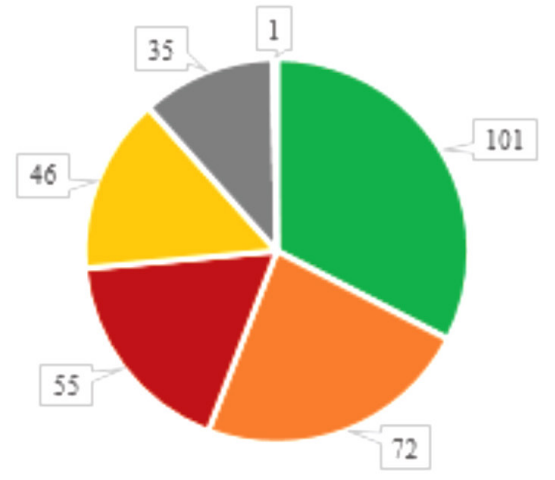

- Natural

" Folklore

- Elementos de interés histórico y cultural

Lugares de realización técnica, centfíca $o$ artistica contemporánea (incluido el turismo astronómico)

- Acontecimientos programados

- Centros o lugares de esparcimiento

Fuente: elaborado con base en SERNATUR, 2012a.

La mayoría de estos recursos turísticos, con una jerarquía importante, se encuentran en la provincia de Elqui y estas "maravillas" se asocian con sitios naturales. En este contexto, se explica el crecimiento del "turismo astronómico" ya que dos de los sitios mencionados anteriormente (Observatorio Cruz del Sur y el Parque Nacional Bosque Fray Jorge, ahora "Reserva Starlight") se relacionan con esta actividad, en la valoración de la bóveda celeste como un recurso turístico, tanto que el primero de ellos fue votado como el número 1 de las maravillas turísticas regionales.

La actividad turística en Chile ha ido en aumento en los últimos decenios. Cada vez más, se ofertan destinos distintos a la capital, Santiago, que es donde concurre la mayor cantidad de turistas, tanto nacionales como extranjeros. El abanico de posibilidades de viaje dentro del país abarca del árido Norte a la Patagonia chilena. En este contexto, en la región de Coquimbo se promueven tipos de actividad turística particulares que le permiten competir con otras en el territorio nacional, uno de ellos es el turismo astronómico.

En 2011, el SERNATUR reportó que de los más de 3 millones de turistas internacionales que llegaron a Chile desde el exterior, el 45\% se dirigió a la región Metropolitana, donde se ubica Santiago; seguida de 
Juan de Dios Páramo Gómez, Álvaro Sánchez Crispín. Estructura territorial del turismo astronómico en la región de Coquimbo, Chile

Valparaíso, con 23\%, Arica con 19\% y, en el lugar décimo, la región de Coquimbo con más de 16 mil visitantes. Esta preferencia territorial es distinta si se consideran los turistas nacionales quienes tienen como destinos preferenciales al litoral de Algarrobo-Santo Domingo, en la región de Valparaíso (12\%); la conurbación Valparaíso-Viña del Mar (10\%); Santiago y la zona alpina de Farellones (8\%) y la región de Coquimbo (5\%). En 2014, el Instituto Nacional de Estadísticas (INE), en la región de Coquimbo, registró la llegada de más de 315 mil personas que incluyen tanto turistas nacionales como extranjeros que, en su mayoría, arriban durante el verano del hemisferio sur, entre enero y febrero.

\section{Infraestructura: la bóveda celeste como recurso turístico}

Una de las circunstancias por las que Chile es considerado como "la capital mundial de la Astronomía" es la cantidad de noches despejadas al año (más de 300). El resultado de tener estos cielos "limpios" no sólo se vincula con las condiciones geográficas y astronómicas del lugar, sino también con la aplicación políticas públicas y marcos legales a los que están sujetas la conservación y la protección de los cielos bajo una medida ambiental.

Los cielos de Chile son predilectos para la observación astronómica, es por ello que Galaz (2001) lo llama "un mirador privilegiado". El Norte Chico de Chile ha logrado una evolución significativa en la Astronomía, desde el avance de esta ciencia en su conocimiento científico hasta proyectos relacionados especialmente con el turismo. El progreso de la Astronomía en Chile se atribuye a la construcción de infraestructura especializada, desde hace más de 50 años; en la actualidad, es el país con el mayor número de observatorios y proyectos astronómicos del mundo, particularmente se localizan en el norte del país: Antofagasta (II Región), Atacama (III Región) y Coquimbo (IV Región). Cabe mencionar que la construcción de observatorios (científicos) se liga, fundamentalmente, con el interés de otros países para el estudio del universo.

Uno de los primeros observatorios astronómicos turísticos del mundo en ser construido es el ubicado en "Cerro Mamalluca", que está en funcionamiento desde 1998. A partir de ese momento, se puede mencionar que nace de manera institucional el "turismo astronómico" en la IV Región, ya que el acceso a este lugar no es restringido y los turistas con poco conocimiento respecto a la Astronomía pueden tratar de entender y mirar con curiosidad lo que compone

194 Revista Geográfica de América Central No 61E (3) Especial CLAG e-ISSN 2215-2563 • Julio-diciembre 2018 • pp. 181-206 Doi: http://dx.doi.org/10.15359/rgac.61-3.10 
la bóveda celeste. El proyecto inició a partir de la donación de equipo por parte del Observatorio Internacional Cerro Tololo (observatorio científico próximo a Cerro Mamalluca); posteriormente, el gobierno de la Municipalidad de Vicuña, junto con el gobierno regional, otorgaron facilidades para construir el observatorio en las cercanías el cerro "Mamalluca". Uno de los motivos por los cuales este observatorio ha logrado ser el referente del turismo en la región es la promoción turística que le ha otorgado SERNATUR y las diversas agencias de viaje que lo posicionan como el más visitado de todos los observatorios astronómicos regionales por parte de turistas a nivel nacional e internacional (Viterbo, op.cit.; Barraza y González, op.cit.).

El SERNATUR cataloga seis observatorios astronómicos turísticos principales en la IV Región, tres son de capital público y tres cuentan con financiamiento privado; cabe señalar que existe otro tipo de forma de observar el cielo a partir de la visita a lugares específicos como la Quebrada de Talca o el Parque Nacional Fray Jorge. En estos sitios, se ofrece llevar a cabo una observación directa de la bóveda celeste sin telescopio, en la que el espectador es testigo del impacto visual que genera el avistamiento del firmamento en la oscuridad.

Los observatorios astronómicos se dividen en dos clases: los de tipo científico y los turísticos. Los primeros tienen como función principal, la investigación del universo; el acceso a ellos, por parte del público en general, está limitado a una cantidad pequeña de personas; se reduce a visitas diurnas programadas con mucha anticipación, una vez a la semana, que permiten recorrer las instalaciones. Estos observatorios poseen los mejores telescopios del mundo y, en el caso de Chile, la mayoría fueron construidos a partir de financiamiento extranjero, por parte de instituciones de Estados Unidos, Brasil, Argentina, Reino Unido y Japón, entre otros.

Por otro lado, se encuentran los observatorios turísticos, a los cuales asisten aficionados que tienen poco conocimiento de Astronomía; no obstante, eso no limita la curiosidad de conocer y observar las estrellas, los planetas $\mathrm{y}$, en general, el firmamento. En esencia, estos sitios se visitan de noche; su objetivo fundamental es el aprendizaje y enseñanza de un nuevo conocimiento al visitante y que, con ello, logre una experiencia significativa en su vida. Un observatorio turístico no se puede comparar con uno científico, ni por su infraestructura, ni por su equipo, ya que es pequeño, tanto en sus instalaciones como en lo referente al tipo de telescopios de los que disponen. 
Juan de Dios Páramo Gómez, Álvaro Sánchez Crispín. Estructura territorial del turismo astronómico en la región de Coquimbo, Chile

Los observatorios astronómicos turísticos ofrecen diferentes visitas que dependen, en gran medida, de la época del año para su funcionamiento; de la disponibilidad de lugares, porque el verano chileno es la temporada con mayor demanda de turistas en la Región; y la hora en la que ofrecen los recorridos, que son, fundamentalmente después del atardecer.

De acuerdo con el catálogo oficial de SERNATUR (2012b), hay once observatorios principales en la región de Coquimbo. No se debe olvidar que existen otros observatorios en esta región, algunos disponen de telescopios propiedad de aficionados en sus casas, y otros no cuentan con estos aparatos, como es el caso del Parque Nacional Fray Jorge en Limarí y las quebradas de Talca, Uchumi, la Viga y Paihuano, en el valle del Elqui.

Así, la distribución de los observatorios astronómicos en la región de Coquimbo muestra una concentración principal en la provincia de Elqui con diez de los once observatorios, y sólo uno en la provincia de Limarí (figura 5); de manera particular, en la comuna de Vicuña se encuentran cinco, tres de ellos científicos (Cerro Tololo, SOAR, Gemini Sur) y dos turísticos (Mamalluca y el Pangue). El resto son dos observatorios científicos (Cerro La Silla y Las Campanas) hacia el norte, en la comuna de La Higuera; uno en La Serena (Cerro Mayu); otro en Andacollo (Collowara); uno más en Paihuano (Cancana) y hacia el sur, el último, en Combarbalá (Cruz del Sur).

La mayoría de las visitas a estos observatorios turísticos se realiza a través de viajes particulares que se contratan en agencias; cada uno incluye el precio de transportación (ida y vuelta), el pago de entrada al observatorio y un snack de cortesía. Los precios aproximados, por persona, en cada tour, rondan los 35 USD, para ir de La Serena a los observatorios Mamalluca y Collowara (60 km de distancia); otros, como el Pangue y el Cancana, se visitan desde la ciudad de Vicuña, el primero por el mismo precio pero con menor distancia recorrida $(20 \mathrm{~km})$ y el último, por 50 USD (60 km de distancia).

Normalmente, en un observatorio turístico se ofrecen las actividades siguientes:

- Proyección o explicación de un video sobre el Sistema Solar y la cosmovisión andina (30 minutos).

- Observación de la bóveda celeste, en terreno, por medio de telescopios pequeños (una hora). 
Juan de Dios Páramo Gómez, Álvaro Sánchez Crispín. Territorial structure of astronomical tourism in the region of Coquimbo, Chile

- Observación de estrellas o planetas en específico, en la cúpula, a través de un telescopio automatizado (30 minutos).

Figura 5. IV Región: ubicación de observatorios astronómicos, científicos y turísticos, 2015

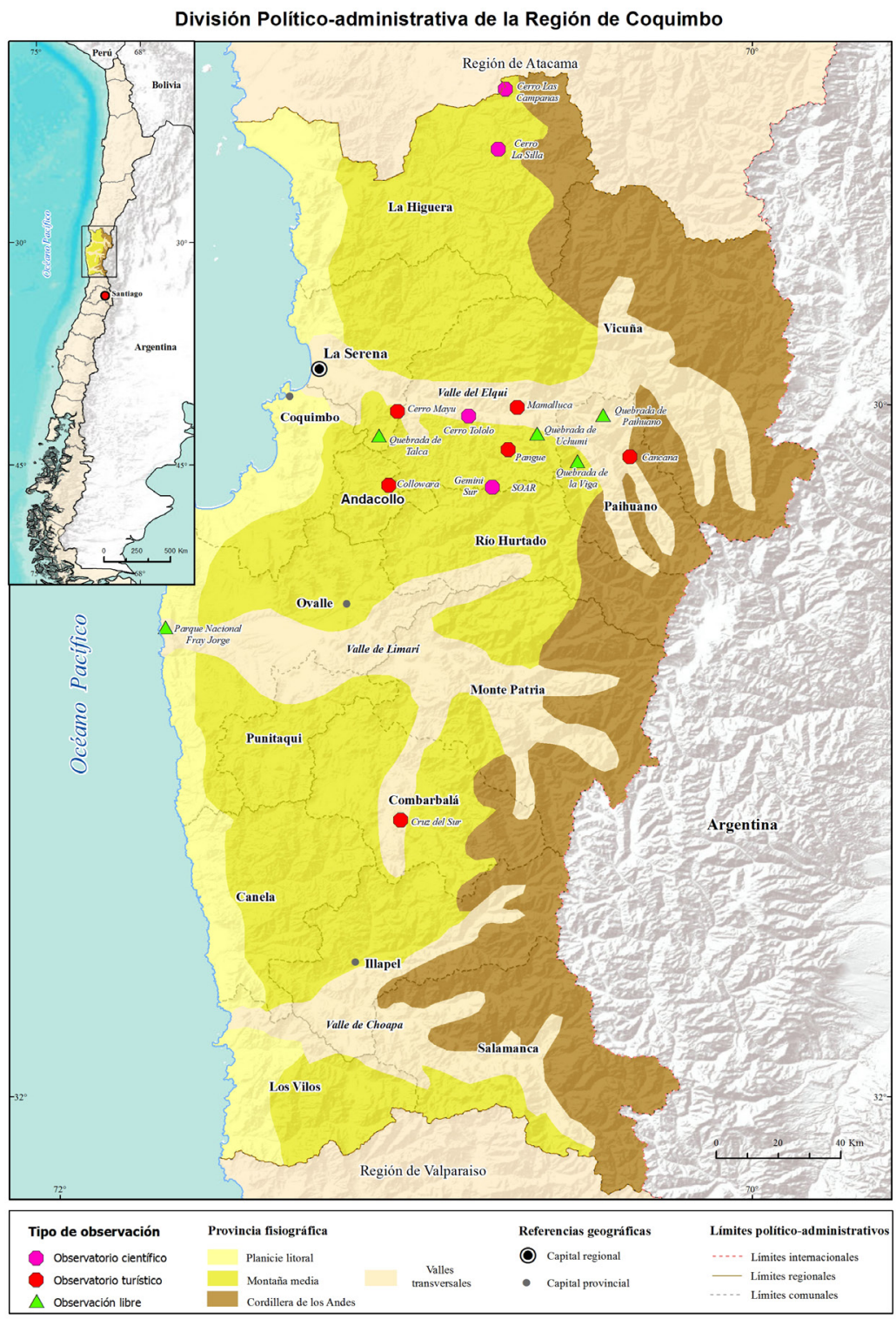

Fuente: elaborado con base en información de observatorios; SERNATUR, 2013. 
Juan de Dios Páramo Gómez, Álvaro Sánchez Crispín. Estructura territorial del turismo astronómico en la región de Coquimbo, Chile

La observación específica en la noche permite contemplar la rotación de la Tierra, al ver un cambio de posición en el movimiento de las estrellas; también, detectar los satélites artificiales en órbita y mirar, a través del telescopio, planetas como Mercurio, Marte, Júpiter y Saturno. Asimismo, se facilita el avistamiento de distintos hechos estelares, por ejemplo, las nubes de Magallanes, la Cruz del Sur y la Vía Láctea, constelaciones con un vínculo en la cosmogonía andina ejemplificadas con una vicuña, y una serpiente, y con animales como el león, el escorpión, y el perro, entre otros. Igualmente, con ayuda de un mapa estelar, se pueden ubicar estrellas y constelaciones específicas en el cielo.

Un punto importante a mencionar dentro de la observación astronómica "aficionada" es que existen aspectos negativos dentro de los observatorios, ya que las instalaciones son totalmente contrarias a las científicas, su función es temática, por ende, algunos sitios como el observatorio "Mamalluca" presenta problemas en su localización geográfica, debido a que se ubica en el piedemonte cordillerano y está a escasos kilómetros de la ciudad de Vicuña, por lo que en algunas ocasiones hay limitaciones que impiden la observación de algunos astros, sobre todo cuando éstos se posicionan en el horizonte; no obstante, no se debe de olvidar que estas instalaciones tienen un fin enfocado hacia la actividad turística, no científica, por lo que la visita a uno de estos centros no deja de ser un hecho significativo en la vida del turista.

Para visitar las instalaciones de un observatorio científico, la dinámica es diferente, desde el registro para la visita por internet, hasta acudir a sus oficinas ubicadas en La Serena, con días de anticipación, para corroborar la asistencia. Llegar a cuatro de los cinco observatorios que permiten las visitas guiadas es complicado, porque es necesario contratar algún servicio especial de traslado hacia los observatorios, o bien, hacerlo a través de transporte privado.

El turismo astronómico no sólo se limita a la observación desde un telescopio, sino también a partir del ojo desnudo, en lugares específicos. Ejemplo de ello son el Parque Nacional Fray Jorge, situado en la provincia de Limarí y que, actualmente, es la primera Reserva Starlight en América, declarada por la UNESCO, en defensa de los cielos nocturnos, y el derecho a la observación estelar. Se trata de proteger y valorizar un recurso natural intangible que significa el cielo, porque éste genera una actividad turística. 
Asimismo, las quebradas tienen una cualidad en la contemplación del universo desde la Tierra, ya que las condiciones geográficas que predominan, y la lejanía de grandes aglomeraciones urbanas, permiten a los visitantes (principalmente chilenos) generar una experiencia de vida asociada con la observación y contemplación del firmamento.

\section{Componentes de la articulación territorial del turismo astronómico}

Los elementos territoriales del turismo astronómico pueden ser resumidos en los siguientes cinco apartados: (Figura 6, en la que se refieren los párrafos siguientes).

Figura 6. Factores y elementos territoriales del turismo astronómico

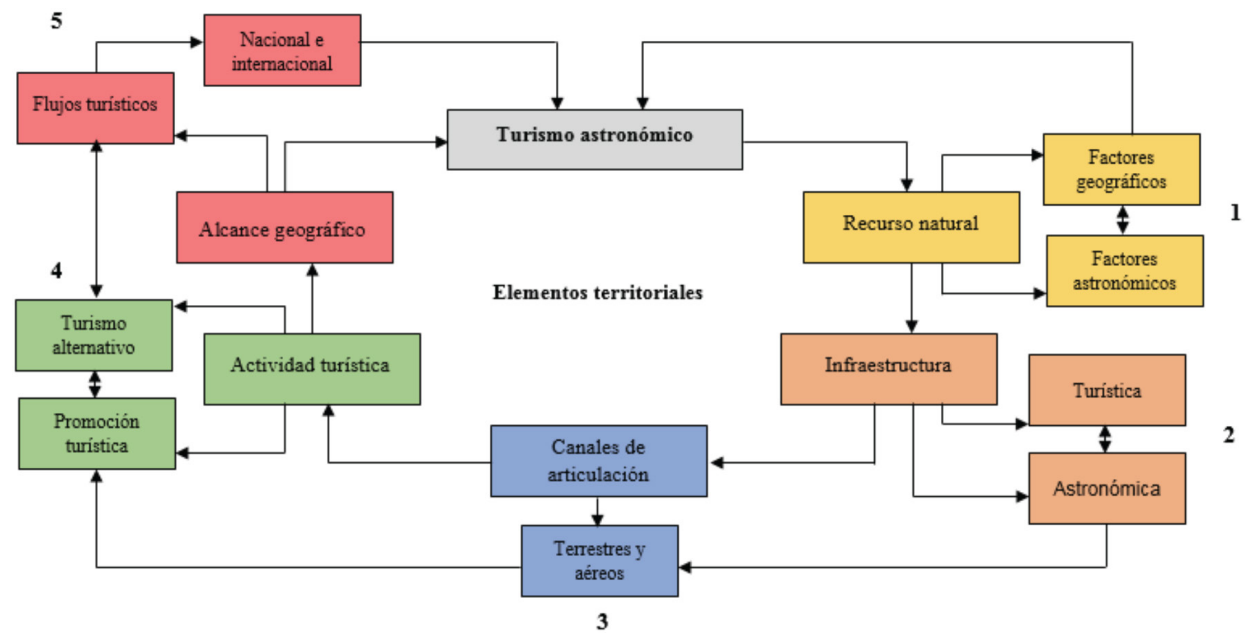

Fuente: elaborado con base en Hernández y Murcio, 2004; Garibay, 2014.

Recurso natural. El firmamento es un recurso que el ser humano valora por su impacto visual y misticismo; además, para que el cielo se encuentre en condiciones perfectas de observación, tienen que coincidir adecuadamente factores geográfico-físicos como la altitud, el relieve, el clima y la precipitación. Entre los elementos astronómicos se encuentra, la posición de la Tierra en diferentes épocas del año, ya que esto influye en poder apreciar distintos ángulos del universo visible.

Infraestructura. Ésta puede ser dividida en dos, la astronómica y la regional-turística; la primera, se asocia con el número de observatorios 
Juan de Dios Páramo Gómez, Álvaro Sánchez Crispín. Estructura territorial del turismo astronómico en la región de Coquimbo, Chile

construidos tanto turísticos, como científicos; igualmente, las vías de comunicación que vinculan a cada observatorio. La segunda es la infraestructura asociada con la economía regional, que incluye el alojamiento, el transporte, y servicios que se ofrecen a los habitantes y al turista.

Canales espaciales de articulación. Son la columna vertebral en la comunicación entre núcleos turísticos; la principal infraestructura para el turismo en la región de Coquimbo es la terrestre (por la accesibilidad y movilidad a los lugares), además también funciona como vía de ingreso al país para turistas extranjeros en el paso "Agua Negra” con más de 22000 personas en 2015 (SERNATUR, 2016). Los canales aéreos son, de igual manera, esenciales por la distancia de los destinos respecto a los múltiples sitios de origen de los visitantes, cabe señalar, que para 2015 arribaron al aeropuerto "La Florida" más de 387000 pasajeros (MOP, 2016); al interconectarse éstos tienen una función como ejes articuladores para la movilidad y transportación del turista, lo que originará flujos turísticos tanto de índole nacional como internacional.

Actividad turística de la región. La promoción del turismo es importante en la región de Coquimbo porque cuenta con un número significativo de recursos que generan distintas variantes: sol y playa, cultural y enológico, entre otros, trascendentales para la economía regional. Como se ha mencionado anteriormente, el turismo astronómico es una actividad incipiente en esta región, su característica principal es la hora en que se realiza (de manera nocturna), de tal manera que es fundamental que se ofrezcan actividades turísticas complementarias durante el día.

Alcance geográfico. Gran parte de los flujos turísticos en la IV Región son de índole nacional, y tienen origen principalmente en la región Metropolitana. El turismo astronómico no escapa a esta tendencia, ya que una proporción mayoritaria de turistas son chilenos, aunque también hay flujos internacionales, cuyos mayores volúmenes se generan desde Alemania, Francia, Estados Unidos, España, Argentina, Canadá y Brasil.

Por tanto, la estructura territorial del turismo astronómico en la IV Región se asocia con el arreglo territorial de los diversos elementos mencionados en la presente investigación; no obstante, una particularidad de esta actividad es que, a diferencia del turismo general, el astronómico además de localizarse puntualmente, tiene una dimensión en la altura, ya que el propósito esencial del turista es lograr una observación del firmamento.

200 Revista Geográfica de América Central No 61E (3) Especial CLAG e-ISSN 2215-2563 • Julio-diciembre 2018 • pp. 181-206 Doi: http://dx.doi.org/10.15359/rgac.61-3.10 
La estructura territorial del turismo astronómico en la IV Región comprende elementos como la Ruta de las Estrellas (establecido por SERNATUR), que atraviesa el valle del Elqui y que abarca las comunas de La Serena, Vicuña y Paihuano (Figura 7); ahí, se encuentra una gran parte de la infraestructura para llevar a cabo el turismo astronómico: los observatorios científicos (Cerro Tololo, Gemini Sur), turísticos (Cerro Mayu, Mamalluca, Pangue, Cancana) y quebradas como las de Talca y Paihuano; si bien existen observatorios y lugares para el desarrollo de esta actividad al norte (observatorios La Silla y Las Campanas) y al sur de la región para observación directa (Parque Nacional Fray Jorge) y mirar el firmamento en uno de los observatorios turísticos más importantes del mundo (Cruz del Sur), la infraestructura turística actual de hospedaje y servicios en estos sitios no tienen la capacidad para la demanda de un mayor número hipotético de turistas (INE, 2014).

La presencia territorial del turismo tiene como referente la articulación de las principales carreteras de la región (ruta 5 y CH-41), concurrentes en la conurbación La Serena-Coquimbo, debido a la cantidad de servicios que presta a los turistas (alojamiento, transporte terrestre y aéreo, tour operadores) que se dirigen especialmente al valle del Elqui; también, se encuentran núcleos secundarios como las ciudades de Ovalle, Salamanca y Los Vilos que cuentan con recursos para el turismo e infraestructura básica. Así, la ruta 5 conecta a la ciudad de La Serena hacia el norte con la ciudad fronteriza de Arica, y al sur con la capital, Santiago; mientras que la ruta $\mathrm{CH}-41$ articula a la capital regional con el valle del Elqui al oriente y finaliza en Agua Negra paso fronterizo con Argentina. 
Juan de Dios Páramo Gómez, Álvaro Sánchez Crispín. Estructura territorial del turismo astronómico en la región de Coquimbo, Chile

Figura 7. IV Región: Estructura territorial del turismo astronómico

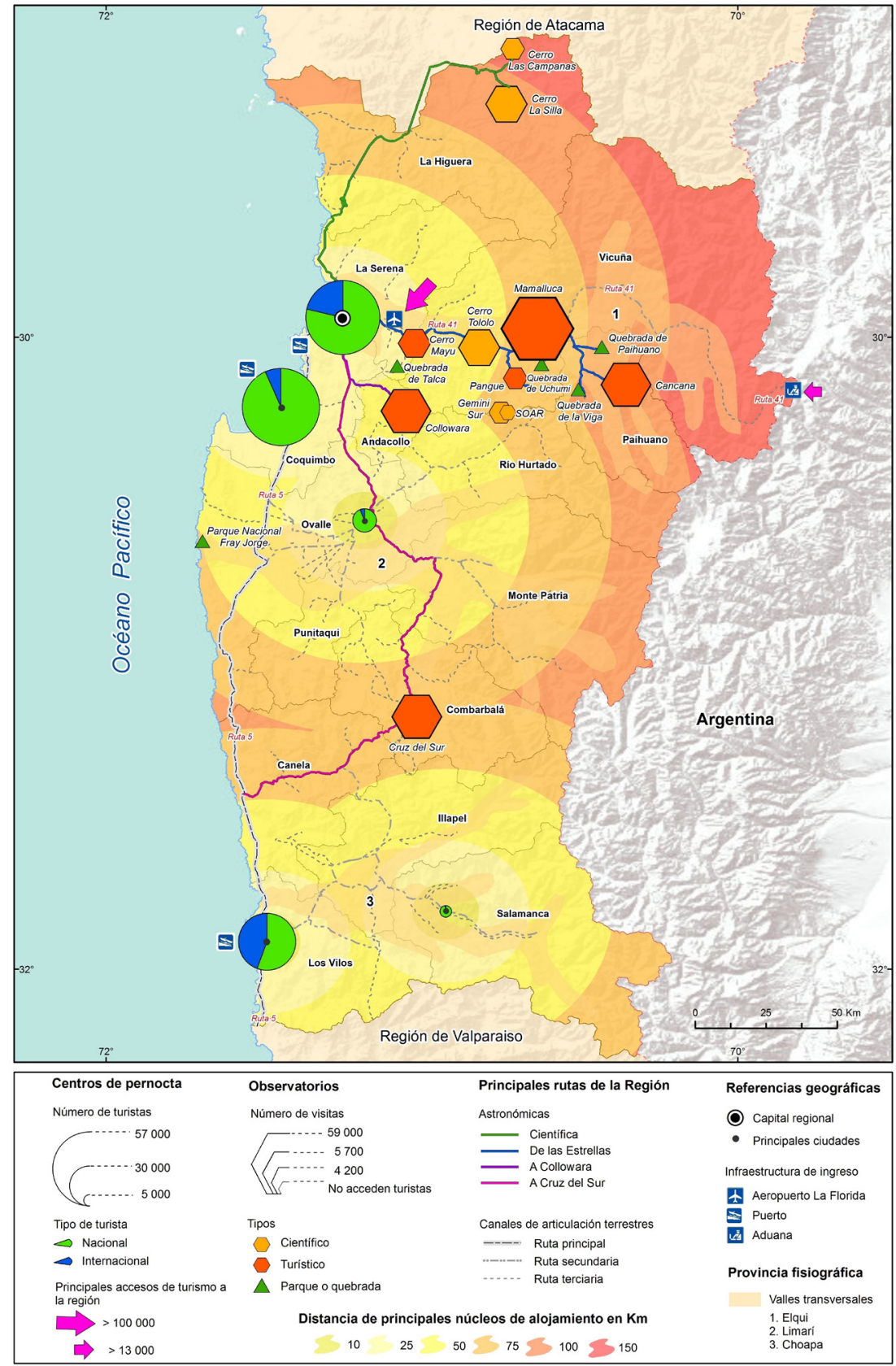

Fuente: elaborado con base en trabajo de campo, 2014; Viterbo, 2012; INE, 2014.

202 Revista Geográfica de América Central No 61E (3) Especial CLAG Este artículo está bajo una licencia e-ISSN 2215-2563・Julio-diciembre 2018・pp. 181-206 Atribución/Reconocimiento-NoCo Doi: http://dx.doi.org/10.15359/rgac.61-3.10 


\section{Conclusiones}

El turismo astronómico es considerado de intereses especiales por ser una actividad no masificada, poco conocida y que demanda particularidades como la espera a que se produzca la noche para poder apreciar los distintos fenómenos que se originan en el firmamento. Este tipo de turismo genera una estructura territorial diferenciable de la del turismo convencional; los recursos que dan sustento al turismo astronómico no están en la superficie sino en la altura y, por tanto, su apreciación es distinta de la que se realiza con respecto a una playa o un glaciar. El impacto visual que se desencadena al observar la bóveda celeste es una experiencia positiva de vida.

La estructura territorial del turismo astronómico tiene un componente pivotal en la región de Coquimbo, el valle del Elqui (Ruta de las Estrellas), en gran medida, por las condiciones geográficas y astronómicas que presenta, como el relieve, el clima, la nula contaminación lumínica, y la lejanía con respecto a grandes centros urbanos. Ahí se concentra y conecta la conurbación La Serena-Coquimbo que funciona como polo económico importante de esta región y como núcleo dominante para los turistas, debido a que en estas ciudades se concentra una gran cantidad de servicios y son paso obligado y sitio de pernocta para quien desea realizar turismo astronómico; por tanto, esta ruta conjunta una parte sustancial de la infraestructura regional turística y de comunicación. Asimismo, es aquí donde se encuentra un gran número de observatorios turísticos y científicos, no sólo de la IV Región, sino del país; además de un parque nacional y quebradas para la observación directa del firmamento.

La actividad turística que representa el turismo astronómico en esta parte de Chile en el futuro mediato pretende ir en aumento; la demanda de turistas por practicarla será mayor, lo que refleja una situación favorable para la economía regional de Coquimbo. No obstante, se debe mencionar que la capacidad de carga en la mayoría de los observatorios representa un aspecto negativo ya que, en ciertas ocasiones, es rebasada (sobre todo en verano), por lo que se podría pensar, en el mediano plazo, en regular la incursión a los observatorios de día y de noche, debido a que el turismo astronómico no se limita a la observación de astros en la oscuridad; además, sería favorable la construcción de más observatorios turísticos, principalmente en el valle del Elqui, lugar con alto potencial para llevar a cabo este tipo de turismo. 
Juan de Dios Páramo Gómez, Álvaro Sánchez Crispín. Estructura territorial del turismo astronómico en la región de Coquimbo, Chile

Para finalizar, existen expectativas en el crecimiento económico de la región con la construcción del proyecto binacional "Túnel de Agua Negra" y el futuro "Corredor Bioceánico" que permitirá la interconexión entre los puertos de Coquimbo y Sao Paulo en Brasil; por todo lo anterior, se prevé un incremento tanto en el comercio como el desplazamiento del turismo masivo en busca de una actividad turística alternativa, lo que podría alterar la dinámica que actualmente tiene para el turismo astronómico en la IV Región.

\section{Referencias}

Barrado, A. (2004). Ordenación territorial y desarrollo turístico. Posibilidades, modelos y esquemas de ordenación territorial del turismo en España. Instituto de Estudios Turísticos, España, en Estudios Turísticos, No. 149, pp. 3 - 22.

Barraza, F. y González, S. (2012). Diseño de planes de acción para integrar el producto turismo astronómico dentro de la oferta turística de la región de Coquimbo. Universidad de La Serena, Chile.

Barrientos, F. y López, S. (2008). Con ojos de gigantes. La observación astronómica en el siglo XXI. Ediciones B. Chile.

Brotherton, B. y B. Himmetoglu (1997). Beyond destinations - special interest tourism, en Anatolia: an International Journal of Tourism and Hospitality Research, Vol. 8, No.3, pp. 11-30.

Cinzano, P., Falchi, F. y Elvidge, D. (2001). The first world atlas of the artificial night sky brightness, Mon. Not. R. Astron. Soc. 328, pp. 689 - 707.

Espinoza, A., Llancaman, M. y Sandoval, H. (2014). Turismo de intereses especiales y Parques nacionales, en Estudios y perspectivas en turismo, Vol. 23, pp. 115 - 130.

Galaz, G. (2001). Astronomía y desarrollo regional: Un cielo lleno de oportunidades, en Rev. Ambiente y Desarrollo, Ambiente hoy, Vol. XVII. No. 3, pp. 38 - 43.

Garibay, A. (2014). Estructura territorial del turismo en el Parque Nacional Volcán Irazú, Costa Rica. Tesis de Licenciatura en Geografía. Facultad de Filosofía y Letras, UNAM, México.

Hernández, C. y Murcio, R. (2004). Estudio de indicadores ONU-Habitat para los observatorios urbanos locales de las ciudades mexicanas, SEDESOL, ONU-Habitat México. 
INE (2011). Informe económico regional, Julio - septiembre, Chile.

IN. (2014). Boletín de alojamiento turístico región de Coquimbo, Diciembre, 2014, Chile.

Kruja, D. y Gjyrezi, A. (2011). The Special Interest Tourism. Development and small regions, en Turizam, Vol. 15, pp. 77 - 89.

Lépez, H. y Torre, G. (2007). Patrimonio cultural astronómico como recurso turístico, en Jornadas Patrimonio y turismo, FFHyA, San Juan, Argentina.

Ministerio de Obras Públicas (MOP), (2016). Aeródromo La Florida de La Serena. Gobierno de Chile.

Newsome, D., S. Moore y R. Dowling (2012). Natural area tourism.

Ecology, impacts and management. Chanel View Publications, 2da edición, Canadá.

Observatorio Astronómico Cruz del Sur (2014). Imágenes del Observatorio Cruz del Sur. Recuperado: [http://observatoriocruzdelsur.cl/ index.php/observatorio/galeria-de-imagenes: 24 de junio de 2015].

OPCC (s/a). Protegiendo los cielos del norte de Chile: Patrimonio ambiental y cultural, Recurso didáctico, Oficina de Protección de la Calidad del Cielo del Norte de Chile, Chile.

Páramo, J. (2015). Estructura territorial del turismo astronómico en la Región de Coquimbo, Chile. Tesis de Licenciatura en Geografía. Facultad de Filosofía y Letras, UNAM, México.

Ramírez, A., Corco, C., Jaque, M. y Lazo, W. (2011). Un sol en el día y millones de soles en la noche: Astronomía en la Región de Coquimbo, Universidad de la Serena, Chile.

Raymond, W. (2013). El cielo ya no puede esperar. Turismo astronómico en el Parque Nacional "El leoncito". Recuperado en: [http://suite101. net/article/el-cielo-ya-no-puede-esperar-a13135\#.VMm6HWiG-So: 15 de abril de 2015].

SERNATUR (2011). Plan para el desarrollo turístico de la región de Coquimbo 2011 - 2014. Servicio Nacional de Turismo, Chile.

SERNATUR (2012a). Atractivos turísticos 2012, Chile.

SERNATUR (2012b). Turismo astronómico, SERNATUR región de Coquimbo, Chile.

SERNATUR (2016). Llegada de turistas extranjeros, agosto 2016. Chile.

SERNATUR / Turismo región de Coquimbo (2013), Conoce la ruta de las

7 maravillas turísticas de la región de Coquimbo, Chile. 
Juan de Dios Páramo Gómez, Álvaro Sánchez Crispín. Estructura territorial del turismo astronómico en la región de Coquimbo, Chile

Trauer, B. (2006). Conceptualizing special interest tourism - frameworks for analysis, en Tourism Management. University of Queensland. Australia, pp. 183 - 200.

Viterbo, A. (2012). La Exposición como medio de promoción y difusión de la Ruta Astronómica de la Cuarta Región, Universidad de Chile.

Weaber, D. (2011). Celestial ecotourism: new horizons in nature-based tourism, en Journal of Ecotourism Vol. 10, No. 1. March, pp. 38 - 45. 